\title{
On the Study of Trigonometric Polynomials Using Strum Sequence
}

\author{
Saiful R. Mondal $\mathbb{D}^{1},{ }^{1}$ Kottakkaran Sooppy Nisar $\mathbb{D}^{2},{ }^{2}$ and Thabet Abdeljawad $\mathbb{C D}^{3,4,5}$ \\ ${ }^{1}$ Department of Mathematics and Statistics, King Faisal University, Al-Hasa 31982, Saudi Arabia \\ ${ }^{2}$ Department of Mathematics, College of Arts and Science, Prince Sattam bin Abdulaziz University, Wadi Aldawaser, \\ 11991, Saudi Arabia \\ ${ }^{3}$ Department of Mathematics and General Sciences, Prince Sultan University, P.O. Box 66833, Riyadh 11586, Saudi Arabia \\ ${ }^{4}$ Department of Medical Research, China Medical University, Taichung 40402, Taiwan \\ ${ }^{5}$ Department of Computer Science and Information Engineering, Asia University, Taichung 40402, Taiwan
}

Correspondence should be addressed to Thabet Abdeljawad; tabdeljawad@psu.edu.sa

Received 21 July 2020; Revised 19 October 2020; Accepted 26 October 2020; Published 21 November 2020

Academic Editor: Efthymios G. Tsionas

Copyright (c) 2020 Saiful R. Mondal et al. This is an open access article distributed under the Creative Commons Attribution License, which permits unrestricted use, distribution, and reproduction in any medium, provided the original work is properly cited.

This article constructs trigonometric polynomials of the sine and cosine whose sums are nonnegative. As an application, those nonnegative trigonometric sums are used to study the geometric properties of complex polynomials in the unit disk. The Strum sequences are used to prove the main outcome.

\section{Introduction}

The trigonometric polynomials with nonnegative sums and its enormous application in various branches of mathematics and science are well known. There are several theories in the literature to find necessary (or/and sufficient) conditions by which

$$
\begin{aligned}
C(\theta) & =\sum_{n=0}^{m} a_{n} \cos (n \theta) \\
S(\theta) & =\sum_{n=1}^{m} a_{n} \sin (n \theta), \quad \theta \in(0, \pi)
\end{aligned}
$$

are nonnegative or positive. This is a century old problem and many mathematicians [1-10] have contributed numerous results. The geometric function theory (univalent functions) has a very close association with the positive trigonometric sums. Both areas have taken and given to each other as evident in the work [11-22]. Here, we intend to present few more results of this interplay.

An analytic function $f$ is univalent in the unit disk $\mathbb{D}=$ $\{z \in \mathbb{C}:|z|<1\}$ if for $z_{1} \neq z_{2}$ implies $f\left(z_{1}\right) \neq f\left(z_{2}\right)$. Let $\mathcal{S}$ be the class of all univalent functions with normalization $f(0)=0=f^{\prime}(0)-1$ and have the series form

$$
f(z)=z+\sum_{n=2}^{\infty} a_{n} z^{n} .
$$

Denote by $\mathscr{A}$, the class of all analytic functions of form (2). A domain $D \in \mathbb{C}$ is said to be starlike with respect to a point $z_{0} \in D$ if the line segment joining any arbitrary chosen points in $D$ with $z_{0}$ lies completely in $D$. The domain $D$ will be convex if it is star-like with respect to all of its points. Analytically, these classes are characterized as

$$
\begin{aligned}
& f \in \mathcal{S}^{*} \Longleftrightarrow \operatorname{Re}\left(\frac{z f^{\prime}(z)}{f(z)}\right)>0, \\
& f \in \mathscr{C} \Longleftrightarrow \operatorname{Re}\left(1+\frac{z f^{\prime \prime}(z)}{f^{\prime}(z)}\right)>0,
\end{aligned}
$$

where $\mathcal{S}^{*}$ and $\mathscr{C}$, respectively, denote the class of star-like and convex functions.

The aim of this article is to construct the star-like polynomials. It is known that partial sum of a univalent function in $\mathbb{D}$ need not be univalent. For example, the Koebe 
function $k(z)=z(1-z)^{-2}$ is an extremal function of many classes in the univalent functions theory defined on $\mathbb{D}$. However, it can be observed that $S_{2}(k, z)=z+2 z^{2}$, the $2^{\text {nd }}$ partial sum of the Taylor series for $k(z)$ is univalent only on the disk $\{z:|z|<1 / 4\}$. Thus, the geometric properties of an analytic function do not inherit to its partial sum. These motivate us to construct the polynomial which are star-like on $\mathbb{D}$.

The following result related to the starlikeness of $f$ is required in sequel.

Lemma 1 (see [23]). If $f^{\prime}$ is typically real in $\mathbb{D}$, that is, $(\operatorname{Im} z)\left(\operatorname{Im} f^{\prime}(z)\right)>0$ for $z \in \mathbb{D}$ and satisfies $\operatorname{Re} f^{\prime}(z)>0$, $z \in \mathbb{D}$, then $f$ is star-like in $\mathbb{D}$.

It is clear from Lemma 1 that the positivity of cosine and sine sums has a significant role to study the star-like polynomials. During this investigation, we observed that, along with other conditions, the monotonicity of $a_{n}$ is pivotal for the positivity or nonnegativity for the sine and cosine sums. This makes us think about what will be the case if we chose $a_{k}$ randomly (especially nonmonotone)? How we can prove the positivity of those trigonometric sums with arbitrary coefficients? Can those positive sums have an application in the study of geometric function theory?

Here, we will provide some affirmative answers for the above questions. The work is motivated from the recent work by Kwong [24], where several trigonometric polynomials with positive sums are given.

\section{Statements of Main Results and Their Consequences}

Now, we will state our main results. The first result will give the positivity of cosine and sine sum with nonmonotonic coefficients.

Theorem 1. For $\theta \in(0, \pi)$, the cosine sum

$$
\begin{aligned}
C_{1}(\theta)= & 1+\frac{\cos (\theta)}{2}+\frac{\cos (2 \theta)}{6}+\frac{\cos (3 \theta)}{4}+\frac{\cos (4 \theta)}{9} \\
& +\frac{\cos (5 \theta)}{8}+\frac{\cos (6 \theta)}{7},
\end{aligned}
$$

and the sine sum

$$
\begin{aligned}
S_{1}(\theta)= & \frac{\sin (\theta)}{2}+\frac{\sin (2 \theta)}{6}+\frac{\sin (3 \theta)}{4}+\frac{\sin (4 \theta)}{9} \\
& +\frac{\sin (5 \theta)}{8}+\frac{\sin (6 \theta)}{7},
\end{aligned}
$$

are positive.

The basic identity $\cos (2 \pi-\theta)=\cos (\theta)$ implies that $C_{1}(\theta)>0$ for all $\theta \in(0,2 \pi)$.

In the sense of complex function theory, the above two positive trigonometric sums can be described as follows. On the boundary of the unit circle $|z|=1$, that is, for $z=e^{i \theta}, \quad 0 \leq \theta \leq 2 \pi, \operatorname{Re}\left(f\left(e^{i \theta}\right)\right)>0$; while $\operatorname{Im}\left(f\left(e^{i \theta}\right)\right)>0$ when $\theta \in(0, \pi)$. Now, by reflection principal, it follows that $\operatorname{Im}\left(f\left(e^{i \theta}\right)\right)<0$ when $\theta \in(\pi, 2 \pi)$. Here,

$$
f(z) \llbracket 1+\frac{z}{2}+\frac{z^{2}}{6}+\frac{z^{3}}{4}+\frac{z^{4}}{9}+\frac{z^{5}}{8}+\frac{z^{6}}{7} .
$$

Denote $\quad g(z):=\int_{0}^{z} f(t) \mathrm{d} t$. Then, clearly $\operatorname{Re} g^{\prime}(z)=\operatorname{Re} f\left(r e^{i \theta}\right)$ and $\operatorname{Im} g^{\prime}(z)=\operatorname{Im} f\left(r e^{i \theta}\right), 0 \leq r<1$. Since $\operatorname{Re}\left(f\left(e^{i \theta}\right)\right)>0$, the minimum principal for harmonic functions yield $\operatorname{Re}\left(f\left(e^{i \theta}\right)\right)>0$; similarly, $\operatorname{Im} g^{\prime}(z)>0$ when $\operatorname{Im} z>0$.

The above fact together with Lemma 1 gives the following result.

Theorem 2. The polynomial

$$
g(z)=z+\frac{z^{2}}{4}+\frac{z^{3}}{18}+\frac{z^{4}}{16}+\frac{z^{5}}{45}+\frac{z^{6}}{48}+\frac{z^{7}}{49},
$$

is star-like with respect to the origin in $\mathbb{D}$.

Theorem 1 can also be validated from Figures 1(a) and 1(b), while starlikeness of $g$ can be seen in Figure 2.

Now, by the same argument as in Theorem 1, several other examples can be constructed by suitable positive cosine and sine sums. Now, we state our second theorem.

Theorem 3. The polynomial

$$
g_{1}(z)=z+\frac{8 z^{2}}{31}+\frac{1}{2400} z^{3}+\frac{5}{3}\left(\frac{\sqrt{3}}{2}-\frac{4}{5}\right) z^{4}
$$

maps the unit disk $\mathbb{D}$ to a star-like domain.

Our third result can be stated as follows.

Theorem 4. The polynomial

$$
h_{n}(z)=z+\sum_{k=1}^{n} \frac{2 z^{2 k+1}}{3(2 k+1)(3 k+1)}+\sum_{k=0}^{n} \frac{2 z^{2 k+2}}{3(2 k+2)(2 k+1)},
$$

maps the unit disk to a star-like domain for $n=1,2$.

Before we give analytical Proof of Theorem 4 in Section 3 , the starlikeness of $h_{1}$ and $h_{2}$ can be seen in Figures 3 and 4 , respectively.

\section{Proof of the Main Results}

Proof of Theorem 1. To prove identity (1), we need to recall the classical identity:

$$
\begin{aligned}
& \cos (\alpha)+\cos (\beta)=2 \cos \left(\frac{\alpha+\beta}{2}\right) \cos \left(\frac{\alpha-\beta}{2}\right), \\
& 1 \pm \cos (n \alpha) \geq 0, \quad \text { for all } \alpha \in[0,2 \pi], n=0,1,2, \ldots
\end{aligned}
$$

This implies 


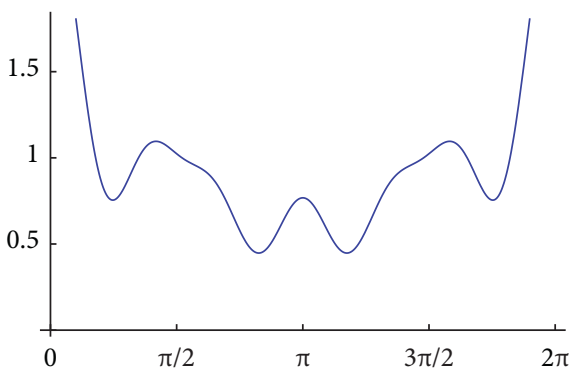

(a)

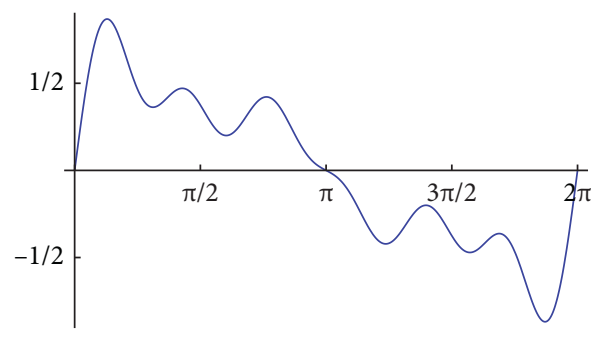

(b)

Figure 1: Image of (a) $C_{1}(\theta)$ and (b) $S_{1}(\theta)$.

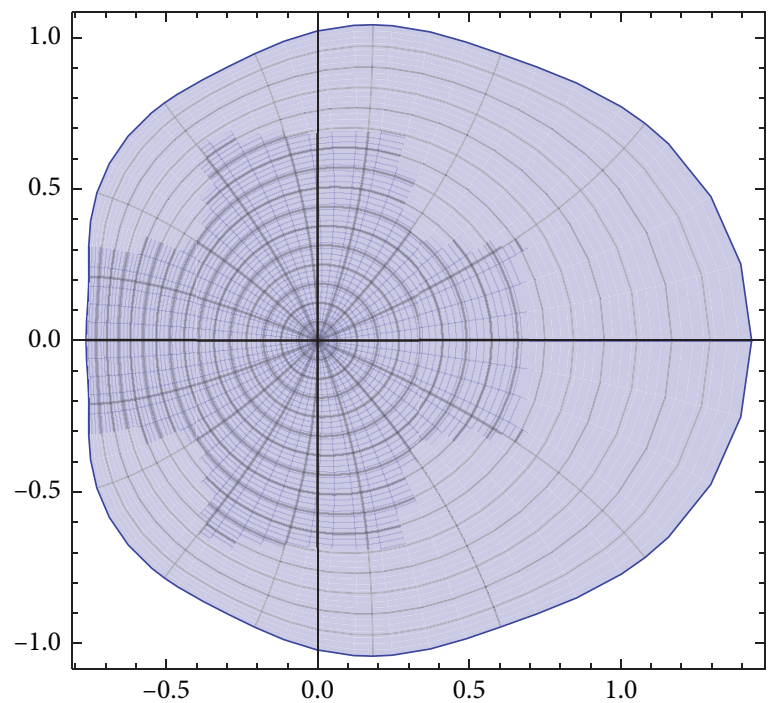

Figure 2: Image of $\mathbb{D}$ under the mapping $g$.

$$
\begin{aligned}
\frac{\cos (\theta)}{2}+\frac{\cos (3 \theta)}{4}+\frac{\cos (5 \theta)}{8}= & \frac{1}{8}(4 \cos (\theta)+2 \cos (3 \theta)+\cos (5 \theta))=\frac{1}{8}(3 \cos (\theta)+(\cos (\theta)+\cos (3 \theta)) \\
& +(\cos (3 \theta)+\cos (5 \theta))) \\
= & \frac{\cos (\theta)}{8}(3+2 \cos (2 \theta)+2 \cos (4 \theta))
\end{aligned}
$$

Now, we can rewrite (1) as

$$
\begin{aligned}
1+ & \frac{\cos (\theta)}{2}+\frac{\cos (2 \theta)}{6}+\frac{\cos (3 \theta)}{4}+\frac{\cos (4 \theta)}{9}+\frac{\cos (5 \theta)}{8}+\frac{\cos (6 \theta)}{7} \\
& =\frac{1}{8}(1+\cos (\theta))(3+2 \cos (2 \theta)+2 \cos (4 \theta))+\left(\frac{5}{8}-\frac{1}{12} \cos (2 \theta)-\frac{5}{36} \cos (4 \theta)+\frac{1}{7} \cos (6 \theta)\right) \\
& =\frac{1}{8}(1+\cos (\theta))\left((1+\cos (2 \theta))^{2}+3 \cos ^{2}(2 \theta)\right)+\left(\frac{131}{504}+\frac{1}{12}(1-\cos (2 \theta))+\frac{5}{36}(1-\cos (4 \theta))+\frac{1}{7}(1+\cos (6 \theta))\right)>0
\end{aligned}
$$

hence proved. 


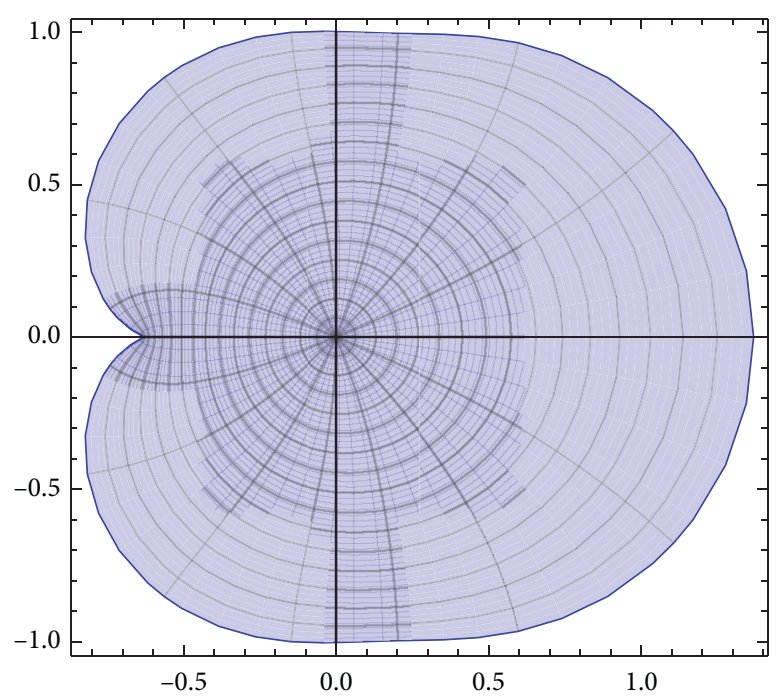

FIgURE 3: Image of $\mathbb{D}$ under the mapping $h_{1}$.

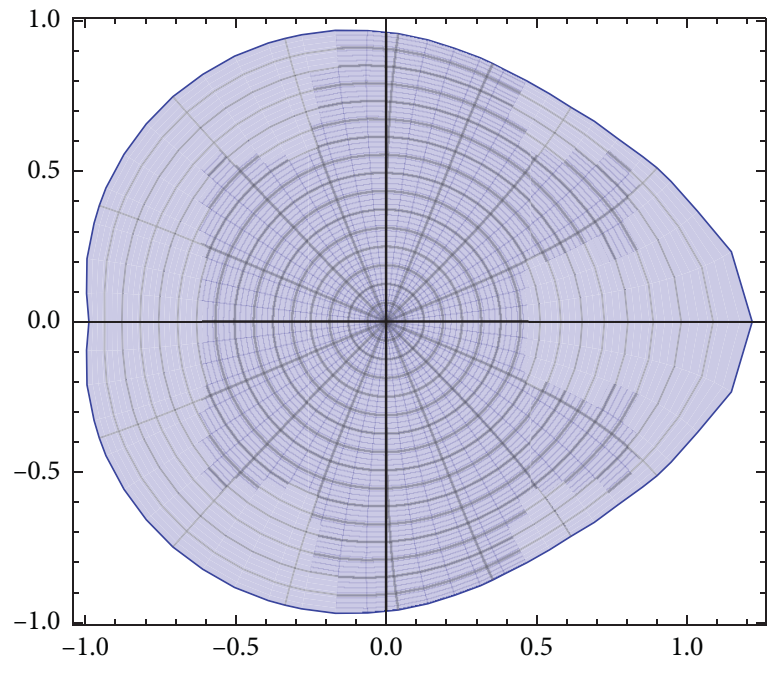

FIgURe 4: Image of $\mathbb{D}$ under the mapping $h_{2}$.

Next, we will prove the positivity of the sine sum in (5). From the well-known trigonometric identities, one can obtain the following identities:

$$
S_{1}(\theta)=\frac{\sin (\theta)}{504}(441+424 \cos (\theta)+378 \cos (2 \theta)+256 \cos (3 \theta)+126 \cos (4 \theta)+144 \cos (5 \theta)) .
$$

It is known that $\sin (\theta)$ is positive for $0<\theta<\pi$, and hence $S_{1}(\theta)>0$ if

$$
\begin{aligned}
& 441+424 \cos (\theta)+378 \cos (2 \theta)+256 \cos (3 \theta)+126 \cos (4 \theta)+144 \cos (5 \theta) \\
& =189+376 \cos (\theta)-252 \cos ^{2}(\theta)-1856 \cos ^{3}(\theta)+1008 \cos ^{4}(\theta)+2304 \cos ^{5}(\theta)>0 .
\end{aligned}
$$


Denote $y:=\cos (\theta)$. Then, clearly $y \in(-1,1)$ and $(14)$ is equivalent to

$$
\begin{aligned}
P_{0}(y)= & 189+376 y-252 y^{2}-1856 y^{3} \\
& +1008 y^{4}+2304 y^{5}>0 .
\end{aligned}
$$

Since $P_{0}(0)=189>0$, inequality (15) holds if $P_{0}$ have no zero in $(-1,1)$. We will prove these facts by constructing Strum sequences to determine the number of zeroes of a polynomial in an open interval.

The Strum sequence for the polynomial $P_{0}$ is given as follows:

$$
\begin{aligned}
& P_{1}(y)=P_{0}^{\prime}(y)=376-504 y-5568 y^{2}+4032 y^{3}+11520 y^{4}, \\
& P_{2}(y)=-\operatorname{rem}\left(P_{0}(y), P_{1}(y)\right)=-\frac{9121}{50}-\frac{15481 y}{50}+\frac{1344 y^{2}}{25}+\frac{20324 y^{3}}{25}, \\
& P_{3}(y)=-\operatorname{rem}\left(P_{1}(y), P_{2}(y)\right)=-\frac{28651198000}{25816561}-\frac{85877316000 y}{25816561}+\frac{36060640800 y^{2}}{25816561}, \\
& P_{4}(y)=-\operatorname{rem}\left(P_{2}(y), P_{3}(y)\right)=-\frac{15786533307854936851}{11287932421064450}-\frac{171856954494801497753 y}{33863797263193350}, \\
& P_{5}(y)=-\operatorname{rem}\left(P_{3}(y), P_{4}(y)\right)=\frac{99571908195813678839431695059474800}{1144025837067465055541881465258369} .
\end{aligned}
$$

Here, by $\operatorname{rem}(P(y), Q(y))$, we mean the remainder of the long division of $P$ by the polynomial $Q$. Now, by Strum theorem the number of roots of $P_{0}$ in $(-1,1)$ is equal to $\sigma(-1)-\sigma(1)$, where $\sigma(a)$ is the number of change of sign by the Strum sequence at the point $a$. From Table 1, it follows that $\sigma(-1)-\sigma(1)=2-2=0$. Thus, $P_{0}$ does not have any zero on $(-1,1)$.

Proof of Theorem 2. Clearly,

$$
g_{1}^{\prime}(z)=1+\frac{16}{31} z+\frac{1}{400} z^{2}+\frac{20}{3}\left(\frac{\sqrt{3}}{2}-\frac{4}{5}\right) z^{3} .
$$

Now, for $z=e^{i \theta}$, it follows that

$$
\begin{aligned}
\operatorname{Reg}_{1}^{\prime}\left(e^{i \theta}\right)=1 & +\frac{16}{31} \cos (\theta)+\frac{1}{400} \cos (2 \theta) \\
& +\frac{20}{3}\left(\frac{\sqrt{3}}{2}-\frac{4}{5}\right) \cos (3 \theta) \\
> & 1-\frac{16}{31}-\frac{1}{400}-\frac{20}{3}\left(\frac{\sqrt{3}}{2}-\frac{4}{5}\right) \approx 0.0412016,
\end{aligned}
$$

and for $0<\theta<\pi$,

$$
\begin{aligned}
\operatorname{Im} g_{1}^{\prime}\left(e^{i \theta}\right) & =\frac{16}{31} \sin (\theta)+\frac{1}{400} \sin (2 \theta)+\frac{20}{3}\left(\frac{\sqrt{3}}{2}-\frac{4}{5}\right) \sin (3 \theta) \\
& =\sin (\theta)\left(\frac{16}{31}+\frac{1}{200} \cos (\theta)+t \frac{20}{3} n\left(\frac{\sqrt{3}}{2}-\frac{4}{5}\right) q\left(4 \cos ^{2}(\theta)-1\right)\right)>\sin (\theta)\left(\frac{16}{31}-\frac{1}{200}-\frac{20}{3}\left(\frac{\sqrt{3}}{2}-\frac{4}{5}\right)\right) \approx 0.0709597 \sin (\theta)>0
\end{aligned}
$$

The result follows from Lemma 1 .

$$
\begin{aligned}
\operatorname{Re}\left(h_{1}^{\prime}\left(e^{i \theta}\right)\right)= & 1+\frac{2}{3} \cos (\theta)+\frac{1}{6} \cos (2 \theta)+\frac{2}{9} \cos (3 \theta) \\
= & \frac{5}{6}+\frac{1}{3} \cos ^{2}(\theta)+\frac{8}{9} \cos ^{3}(\theta) \\
= & \frac{5}{18}+\frac{\cos ^{2}(\theta)}{3}(1+\cos (\theta)) \\
& +\frac{5}{9}\left(1+\cos ^{3}(\theta)\right)>0 .
\end{aligned}
$$

for $\theta \in(0,2 \pi)$.

Clearly, for $n=1$, identity (20) gives
Similarly, for $n=1$, we have 
TABle 1: Change the signs of Strum sequence for $P_{0}$.

\begin{tabular}{lcc}
\hline & $y=-1$ & $y=1$ \\
\hline$P_{0}(y)$ & + & + \\
$P_{1}(y)$ & - & + \\
$P_{2}(y)$ & + & - \\
$P_{3}(y)$ & + & - \\
$P_{4}(y)$ & + & + \\
$P_{5}(y)$ & + & + \\
\hline
\end{tabular}

TABle 2: Change the signs of Strum sequence for $Q$.

\begin{tabular}{lccc}
\hline & $y=-1$ & $y=1$ \\
\hline$Q(y)$ & + & + \\
$Q_{1}(y)$ & - & + \\
$Q_{2}(y)$ & - & - \\
$Q_{3}(y)$ & + & - \\
$Q_{4}(y)$ & + & + \\
\hline
\end{tabular}

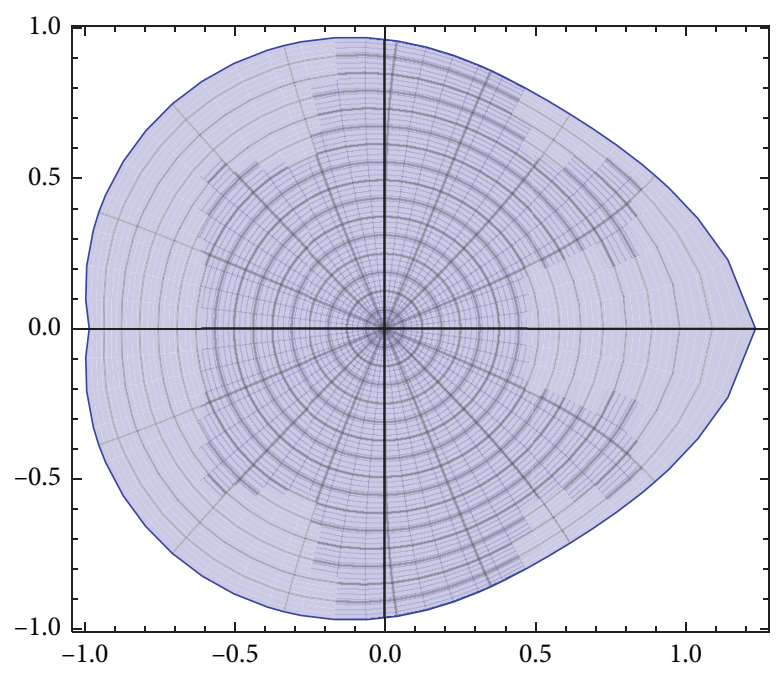

FIGURE 5: Image of $\mathbb{D}$ under the mapping $h_{28}$.

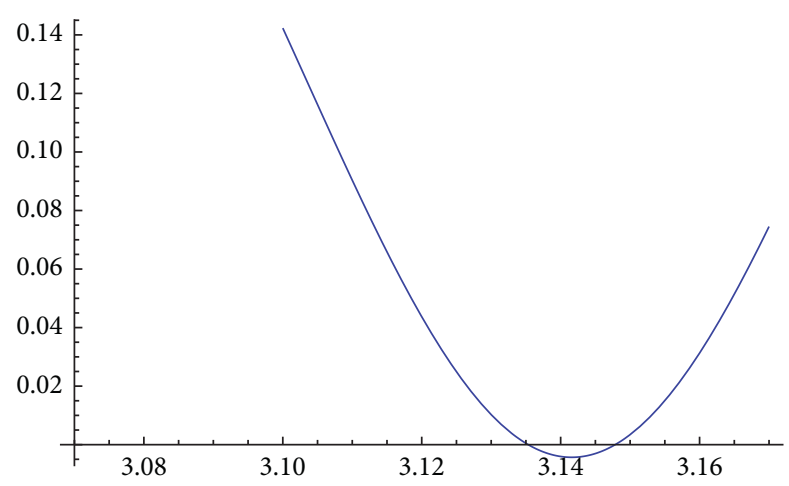

Figure 6: Image of $\operatorname{Re}\left(h_{28}^{\prime}\left(e^{i \theta}\right)\right), 3.1 \leq \theta \leq 3.17$. 


$$
\begin{aligned}
\operatorname{Im}\left(h_{1}^{\prime}\left(e^{i \theta}\right)\right) & =\frac{2}{3} \sin (\theta)+\frac{1}{6} \sin (2 \theta)+\frac{2}{9} \sin (3 \theta) \\
& =\frac{\sin (\theta)}{9}\left(4+3 \cos (\theta)+8 \cos ^{2}(\theta)\right) .
\end{aligned}
$$

If $\theta \in(0, \pi)$, then (22) implies that $\operatorname{Im}\left(h_{2}^{\prime}\left(e^{i \theta}\right)\right)>0$, while if $\theta \in(\pi, 2 \pi)$, then $\operatorname{Im}\left(h_{2}^{\prime}\left(e^{i \theta}\right)\right)<0$. Thus, we can conclude that $\operatorname{Im}(z) \operatorname{Im}\left(h_{2}^{\prime}(z)\right)>0$. Finally, the conclusion follows from Lemma 1.

Next, we will consider the case for $n=2$. In this case,

$$
\begin{aligned}
\operatorname{Re}\left(h_{2}^{\prime}\left(e^{i \theta}\right)\right)= & 1+\frac{2}{3} \cos (\theta)+\frac{1}{6} \cos (2 \theta)+\frac{2}{9} \cos (3 \theta)+\frac{2}{21} \cos (4 \theta)+\frac{2}{15} \cos (5 \theta)=\frac{5}{6}+\frac{1}{3} \cos ^{2}(\theta)+\frac{8}{9} \cos ^{3}(\theta) \\
& +\frac{2}{21} \cos (4 \theta)+\frac{2}{15} \cos (5 \theta) \\
= & \frac{31}{630}+\frac{\cos ^{2}(\theta)}{3}(1+\cos (\theta))+\frac{5}{9}\left(1+\cos ^{3}(\theta)\right)+\frac{2}{21}(1+\cos (4 \theta))+\frac{2}{15}(1+\cos (5 \theta))>0 .
\end{aligned}
$$

Similarly,

$$
\begin{aligned}
\operatorname{Im}\left(h_{1}^{\prime}\left(e^{i \theta}\right)\right) & =\frac{2}{3} \sin (\theta)+\frac{1}{6} \sin (2 \theta)+\frac{2}{9} \sin (3 \theta)+\frac{2}{21} \sin (4 \theta)+\frac{2}{15} \sin (5 \theta) \\
& =\frac{\sin (\theta)}{315}(322+165 \cos (\theta)+224 \cos (2 \theta)+60 \cos (3 \theta)+84 \cos (4 \theta)) \\
& =\frac{\sin (\theta)}{315}\left(182-15 \cos (\theta)-224 \cos ^{2}(\theta)+240 \cos ^{3}(\theta)+672 \cos ^{4}(\theta)\right)
\end{aligned}
$$

It is well known that $\sin (\theta)$ is positive for $\theta \in(0, \pi)$. Thus, it is enough to show that

$$
\begin{aligned}
182 & -15 \cos (\theta)-224 \cos ^{2}(\theta)+240 \cos ^{3}(\theta) \\
& +672 \cos ^{4}(\theta)>0,
\end{aligned}
$$

which is equivalent to prove

$$
Q(y)=182-15 y-224 y^{2}+240 y^{3}+672 y^{4}>0 .
$$

$$
Q_{1}(y)=Q^{\prime}(y)=-15-448 y+720 y^{2}+2688 y^{3}
$$

Next to form the Strum sequence, we need to find the remainder of $Q(y)$ and $Q_{1}(y)$ as

$$
Q_{2}(y)=-\operatorname{rem}\left(Q(y), Q_{1}(y)\right)=-\frac{40843}{224}+\frac{5 y}{4}+\frac{1793 y^{2}}{14}
$$

Since $Q(0)=182>0$, we need to prove that $Q(y)$ does not have any zero in $[-1,1]$. We apply the Strum theorem for this purpose. A differentiation of (26) gives and continuing, similar to the Strum theorem, we have

$$
\begin{aligned}
& Q_{3}(y)=-\operatorname{rem}\left(Q_{1}(y), Q_{2}(y)\right)=-\frac{284283300}{292259}-\frac{10840870880}{3214849} y, \\
& Q_{4}(y)=-\operatorname{rem}\left(Q_{2}(y), Q_{3}(y)\right)=\frac{14441992188069699847}{83946058169165696} .
\end{aligned}
$$

The change of sign of the Strum sequence $\left\{Q, Q_{1}, Q_{2}, Q_{3}, Q_{4}\right\}$, respectively, at $y=-1$ and $y=1$ can be seen in Table 2. Since $\sigma(-1)-\sigma(1)=2-2=0$, the polynomial $Q$ does not have any zero on $(-1,1)$.

Finally, we have $\operatorname{Im}\left(h_{2}^{\prime}\left(e^{i \theta}\right)\right)>0$ for all $\theta \in(0, \pi)$, and by Lemma 1 , it can be concluded that $h_{2}(\mathbb{D})$ is star-like with respect to the origin.

\section{Concluding Remark}

From the above three examples, it can be concluded that the trigonometric sine and cosine sum can also be positive with some nonmonotone coefficients.

In Theorem 4 , though we prove that $h_{n}$ is star-like for $n=1,2$, but the graphical experiment Figure 5 shows that $h_{n}$ 
is star-like for all $n \in \mathbb{N}$. However, $\operatorname{Re}_{n}^{\prime}\left(e^{i \theta}\right)$ is negative for some range of $\theta$ when $n$ is larger (greater than 28, see Figure 6). Thus, our method is not applicable on $h_{n}$ for larger $n$.

The results in this article indicate that several polynomials can be constructed which maps unit disk to a star-like domain by using positivity of cosine and sine sum.

\section{Data Availability}

No data were used in this study.

\section{Conflicts of Interest}

The authors declare that they have no conflicts of interest.

\section{Acknowledgments}

T. Abdeljawad would like to thank Prince Sultan University for funding this work through research group Nonlinear Analysis Methods in Applied Mathematics (NAMAM) group no. RG-DES-2017-01-17.

\section{References}

[1] H. Alzer and S. Koumandos, "Sharp inequalities for trigonometric sums," Mathematical Proceedings of the Cambridge Philosophical Society, vol. 134, no. 1, pp. 139-152, 2003.

[2] H. Alzer and S. Koumandos, "A sharp bound for a sine polynomial," Colloquium Mathematicum, vol. 96, no. 1, pp. 83-91, 2003.

[3] H. Alzer and S. Koumandos, "Inequalities of Fejér-Jackson type," Monatshefte für Mathematik, vol. 139, no. 2, pp. 89-103, 2003.

[4] H. Alzer and S. Koumandos, "Companions of the inequalities of Fejér-Jackson and young," Analysis Mathematica, vol. 31, no. 2, pp. 75-84, 2005.

[5] H. Alzer and S. Koumandos, "A new refinement of Young's inequality," Proceedings of the Edinburgh Mathematical Society, vol. 50, no. 2, pp. 255-262, 2007.

[6] R. Askey and J. Steinig, "Some positive trigonometric sums," Transactions of the American Mathematical Society, vol. 187, p. 295, 1974.

[7] R. Askey and G. Gasper, "Positive Jacobi polynomial sums, II," American Journal of Mathematics, vol. 98, no. 3, pp. 709-737, 1976.

[8] A. S. Belov, "Examples of trigonometric series with nonnegative partial sums. (Russian)," Sbornik: Mathematics, vol. 186, pp. 21-46, 1995.

[9] G. Brown and E. Hewitt, "A class of positive trigonometric sums," Mathematische Annalen, vol. 268, no. 1, pp. 91-122, 1984.

[10] G. Brown, K.-Y. Wang, and D. C. Wilson, "Positivity of some basic cosine sums," Mathematical Proceedings of the Cambridge Philosophical Society, vol. 114, no. 3, pp. 383-391, 1993.

[11] S. Koumandos, On Certain Gegenbauer Polynomial Sums, Analysis and its Applications, Allied Publishers, New Delhi, India, 2000.

[12] S. Koumandos, "An extension of Vietoris's inequalities," The Ramanujan Journal, vol. 14, no. 1, pp. 1-38, 2007.

[13] S. Koumandos and S. Ruscheweyh, "Positive Gegenbauer polynomial sums and applications to starlike functions," Constructive Approximation, vol. 23, no. 2, pp. 197-210, 2006.
[14] S. Koumandos and S. Ruscheweyh, "On a conjecture for trigonometric sums and starlike functions," Journal of Approximation Theory, vol. 149, no. 1, pp. 42-58, 2007.

[15] S. Koumandos and M. Lamprecht, "On a conjecture for trigonometric sums and starlike functions, II," Journal of Approximation Theory, vol. 162, no. 5, pp. 1068-1084, 2010.

[16] S. R. Mondal and A. Swaminathan, "On the positivity of certain trigonometric sums and their applications," Computers \& Mathematics with Applications, vol. 62, no. 10, pp. 3871-3883, 2011.

[17] S. R. Mondal and A. Swaminathan, "Stable functions and extension of Vietoris' theorem," Results Math, vol. 62, no. 1-2, pp. 33-51, 2012.

[18] S. Ruscheweyh, "On the Kakeya-Eneström theorem and gegenbauer polynomial sums," SIAM Journal on Mathematical Analysis, vol. 9, no. 4, pp. 682-686, 1978.

[19] S. Ruscheweyh and L. Salinas, "On starlike functions of order $\lambda \in[1 / 2,1)$," Annales Universitatis Mariae Curie-Sklodowska Section A, vol. 54, pp. 117-123, 2000.

[20] S. Ruscheweyh and L. Salinas, "Stable functions and Vietoris' theorem," Journal of Mathematical Analysis and Applications, vol. 291, no. 2, pp. 596-604, 2004.

[21] P. Sangal and A. Swaminathan, "Starlikeness of Gaussian hypergeometric functions using positivity techniques," Bulletin of the Malaysian Mathematical Sciences Society, vol. 41, no. 1, pp. 507-521, 2018.

[22] P. Sangal and A. Swaminathan, "Geometric properties of Cesàro averaging operators," Journal of Complex Analysis, vol. 2017, p. 9, Article ID 6584584, 2017.

[23] S. Ruscheweyh, "Coefficient conditions for starlike functions," Glasgow Mathematical Journal, vol. 29, no. 1, pp. 141-142, 1987.

[24] M. K. Kwong, "Nonnegative trigonometric polynomials, Sturm's theorem, and symbolic computation," https://arxiv. org/abs/1402.6778. 